towards future developments and alternatives that may supersede the drug eluting stent hegemony.

Conflict of Interest None

\section{A REVIEW OF LEFT MAIN STEM PERCUTANEOUS CORONARY INTERVENTION AT A TERTIARY CENTRE IN THE UNITED KINGDOM}

${ }^{1}$ Anenta Ramakrishnan, ${ }^{2}$ Claudia Cosgrove, ${ }^{2}$ Natalia Briceno, ${ }^{2}$ James Spratt. ${ }^{1} / m p e r i a l$ College London, London, UK; ${ }^{2}$ St George's University Hospitals NHS Foundation Trust

10.1136/heartjnl-2021-BCS.77

Introduction The role of percutaneous coronary intervention (PCI) in left main stem (LMS) disease continues to evolve with advances in stent technology, adjuncts such as intracoronary imaging, calcium modification techniques and left ventricular support devices. Moreover, with changes in the demographics of the UK population and of the patient population presenting with left main coronary artery disease, the applicability of findings from historic clinical trials is uncertain.

Aim We set out to review our routine clinical practice at our tertiary PCI centre. We aimed to compare our practice to contemporary best practice guidelines and to identify a process for on-going audit and improvement.

Methods We performed a retrospective review of all procedures coded for 'Percutaneous Coronary Intervention Left Main Stem' at our centre from 1st December 2018 to 1st June 2019. Coronary angiogram reports and electronic notes for each patient were reviewed. Angiographic images and intracoronary imaging were by two operators.

Results Thirty cases were identified, for twenty-nine individual patients. The average age of patient undergoing LMS PCI was 74 years old. 24 patients were male, 5 patients were female. 28 patients had greater than two co-morbidities, including chronic kidney disease (14 patients) and type 2 diabetes mellitus (11 patients). Nearly two thirds of patients had known left ventricular systolic dysfunction $(n=17)$. The majority of cases $(n=12)$ were for acute coronary syndrome, including 8 for ST-elevation myocardial infarction (STEMI) and/or out of hospital cardiac arrest. The remainder were elective cases for stable angina where optimal medical therapy had failed to relieve symptoms and surgery was not deemed appropriate. Intracoronary imaging was recorded in 24 cases, the majority of which $(n=23)$ employed intravascular ultrasound (IVUS). However, only four cases had documented minimum luminal area (MLA) or minimum stent area (MSA). Thirteen cases required calcium modification, including by cutting balloon $(n=6)$, intravascular lithotripsy $(n=7)$, and rotational atherectomy $(n=1)$.Sixteen cases had distal left main bifurcation disease, and bifurcation PCI techniques included provisional 1stent $(n=6)$, provisional 2-stent $(n=8)$, Culotte $(n=7)$ and $T$ and small protrusion (TAP) stenting. There were four in-hospital deaths, all in patients presenting with STEMI. One patient had a cardiac cause or procedural-related cause for re-presentation to our centre before 1st December 2019 (at least 6 months post-procedure). This presentation was related to heart failure and the patient did not require revascularisation.

Conclusions The case and coronary complexity of patients undergoing left main stem PCI is very high in our centre. We found that the use of intracoronary imaging was not always well documented and available for retrospective review. In view of the high number of cases requiring calcium modification and the high number of cases that included distal left main bifurcation disease, we have taken steps to support the mandated use of intracoronary imaging in left main stem disease within the coronary catheter laboratory team, including educational training and technical support.

Conflict of Interest None

\section{Allied health professionals/Nursing/Health scientists}

\section{AUDIT OF ECHOCARDIOGRAPHIC PROBABILITY OF PULMONARY HYPERTENSION IN A REAL WORLD POPULATION REFERRED TO A SPECIALIST CENTRE}

${ }^{1}$ Oliver Slegg, ${ }^{1}$ James Willis, ${ }^{2}$ Ciara Gibson, ${ }^{2}$ Aidan Kendler-Rhodes, ${ }^{3}$ Fiona Wilkinson, ${ }^{1}$ Jennifer Rossdale, ${ }^{1} \mathrm{Pia}$ Charters, ${ }^{4}$ Ben Hudson, ${ }^{1}$ Rob Mackenzie Ross, ${ }^{1} J$ ohn Pauling, ${ }^{1} J a c o b$ Easaw, ${ }^{4}$ Kevin Carson, ${ }^{4}$ Sri Raveen Kandan, ${ }^{1}$ Graham Robinson, 'Jay Suntharalingam, 'Daniel Auqustine. 'Royal United Hospitals Bath NHS Foundation Trust, Bath, UK; ${ }^{2}$ University of Bristol; ${ }^{3}$ Manchester Metropolitan University; ${ }^{4}$ RUH Bath

\subsection{6/heartjnl-2021-BCS.78}

Introduction Transthoracic echocardiography (TTE) is an established screening tool used in the assessment of suspected Pulmonary Hypertension (PH). There is evidence to suggest Doppler estimates of Pulmonary Artery Systolic Pressure (PASP) are inaccurate compared to Right Heart Catheter (RHC) measures. Therefore, the ESC and BSE recommend a multi parameter assessment of TTE probability of PH. This retrospective audit sought to evaluate the effectiveness of the BSE/ESC TTE probability algorithm in detecting $\mathrm{PH}$ in a real world cohort referred to a shared care $\mathrm{PH}$ centre.

Method Between 2010 and 2019, a total of 310 patients referred for initial assessment of PH underwent TTE followed by RHC (median interval $31 \pm 30$ days). PH TTE probabilities were calculated following BSE/ESC guidance. Bland-Altman analysis was used to investigate the accuracy of Doppler estimates of PASP compared to RHC.

Results The mean sample age was $67 \pm 14$ years (62\% female). Seventy-six percent $(n=235)$ had PH $(\mathrm{mPAP} \geq 25 \mathrm{mmHg})$ (average mPAP $42.8 \pm 11.7 \mathrm{mmHg}), \quad 26 \% \quad(\mathrm{n}=80)$ having CTEPH. Doppler estimates of PASP tended to underestimate true PASP (bias $-3.7 \pm 15.2 \mathrm{mmHg}$ ) with wide limits of

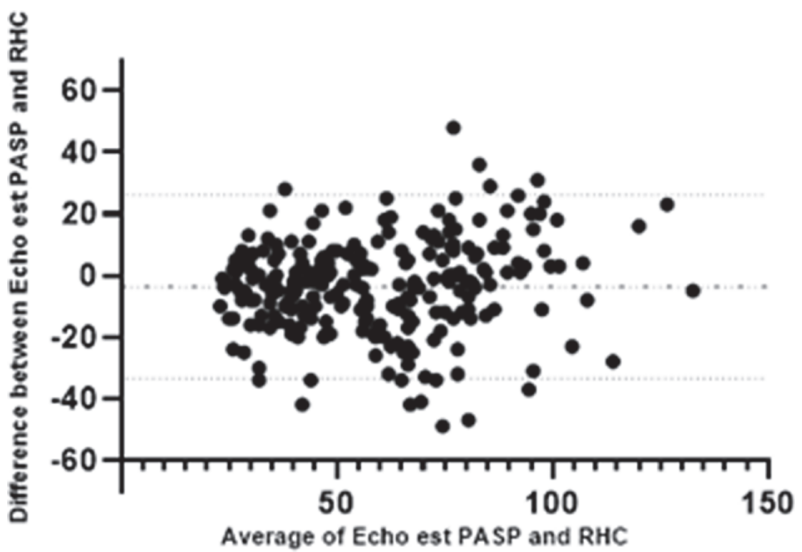

Abstract 78 Figure 1 Bland-Altman plot of echocardiographic estimates of PASP $(n=239)$ compared to RHC measurements 